\title{
Explicit formulas for OPUC and POPUC associated with measures which are simple modifications of the Lebesgue measure
}

\author{
Cleonice F. Bracciali ${ }^{\mathrm{a}, *}$, Jairo S. Silva ${ }^{\mathrm{b}, \mathrm{c}}$, A. Sri Ranga ${ }^{\mathrm{a}}$ \\ a Departamento de Matemática Aplicada, IBILCE, UNESP - Universidade Estadual Paulista, São José do Rio Preto, SP, 15054-000, Brazil \\ b Departamento de Matemática, Universidade Federal do Maranhão, São Luís, MA, 65080-805, Brazil \\ ' Pós-Graduação em Matemática, IBILCE, UNESP - Universidade Estadual Paulista, São José do Rio Preto, SP, 15054-000, Brazil
}

\section{A R T I C L E I N F O}

\section{MSC:}

$42 \mathrm{CO} 5$

$33 C 47$

Keywords:

Orthogonal polynomials on the unit circle

Para-orthogonal polynomials

Chain sequences

\begin{abstract}
A B S T R A C T
We consider nontrivial probability measures, obtained as simple modifications of the Lebesgue measure, which include mass points at $z=1$ and $z=i$. The orthogonal polynomials, para-orthogonal polynomials and Toeplitz matrices associated with these measures are presented.
\end{abstract}

(c) 2015 Elsevier Inc. All rights reserved.

\section{Introduction}

Orthogonal polynomials on the unit circle (OPUC), $S_{n}, n \geq 0$, which also bear the name of Szegö polynomials, can be defined by

$$
\int_{\mathcal{C}} \bar{\zeta}^{j} S_{n}(\zeta) d \psi(\zeta)=\int_{0}^{2 \pi} e^{-i j \theta} S_{n}\left(e^{i \theta}\right) d \psi\left(e^{i \theta}\right)=0, \quad 0 \leq j \leq n-1, \quad n \geq 1,
$$

where $S_{n}$ is a polynomial of exact degree $n$ and $\psi(\zeta)=\psi\left(e^{i \theta}\right)$ is a nontrivial measure supported on the unit circle $\mathcal{C}=\{\zeta=$ $\left.e^{i \theta}: 0 \leq \theta \leq 2 \pi\right\}$. If $\kappa_{n}^{-1}=\left\|S_{n}\right\|=\left\{\int_{\mathcal{C}}\left|S_{n}(\zeta)\right|^{2} d \psi(\zeta)\right\}^{1 / 2}$, then $\kappa_{n}>0$ and the orthonormal polynomials on the unit circle are $s_{n}(z)=\kappa_{n} S_{n}(z), n \geq 0$.

The polynomials $S_{n}, n \geq 0$, considered as monic polynomials, satisfy the so called forward and backward recurrence relations, respectively,

$$
\begin{aligned}
& S_{n}(z)=z S_{n-1}(z)-\bar{\alpha}_{n-1} S_{n-1}^{*}(z), \\
& S_{n}(z)=\left(1-\left|\alpha_{n-1}\right|^{2}\right) z S_{n-1}(z)-\bar{\alpha}_{n-1} S_{n}^{*}(z), \quad n \geq 1,
\end{aligned}
$$

where $\alpha_{n-1}=-\overline{S_{n}(0)}$ and $S_{n}^{*}(z)=z^{n} \overline{S_{n}(1 / \bar{z})}$ denotes the reversed (reciprocal) polynomial of $S_{n}$. The numbers $\alpha_{n}$, in recent years, have been referred to as Verblunsky coefficients. It is known that these coefficients are such that $\left|\alpha_{n}\right|<1, n \geq 0$, as well as that

\footnotetext{
The first and third authors are supported by funds from CNPq (\#401891/2013-5, \#475502/2013-2) and FAPESP (\#09/13832-9, \#14/22571-2) of Brazil. The second author is supported by grant from CAPES of Brazil.

* Corresponding author. Tel.: +55 17 32212213, fax: +55 1732212203.

E-mail addresses: cleonice@ibilce.unesp.br, cleonice.bracciali@gmail.com (C.F. Bracciali), jairo.santos@ufma.br (J.S. Silva), ranga@ibilce.unesp.br (A. Sri Ranga).
} 
OPUC and the associated measure are completely characterized by the Verblunsky coefficients $\left\{\alpha_{n}\right\}_{n=0}^{\infty}$. See Ismail [7], Simon $[8,9]$ and Szegő [11].

Para-orthogonal polynomials on the unit circle (POPUC) associated with the sequence of OPUC $\left\{S_{n}\right\}_{n=0}^{\infty}$ can be given by

$$
z S_{n-1}(z)-\tau_{n} S_{n-1}^{*}(z), \quad n \geq 1,
$$

where $\left\{\tau_{n}\right\}_{n=1}^{\infty}$ is any sequence of complex numbers such that $\left|\tau_{n}\right|=1$. Different from $S_{n}$ which has all its $n$ zeros within the open unit disk, the polynomial $z S_{n-1}(z)-\tau_{n} S_{n-1}^{*}(z)$ has all its $n$ zeros simple and lying on the unit circle.

Another entity that plays an important role in the theory of OPUC are the Toeplitz matrices defined by

$$
\mathbf{T}_{n}=\left[\begin{array}{cccc}
\mu_{0} & \mu_{-1} & \cdots & \mu_{-n} \\
\mu_{1} & \mu_{0} & \cdots & \mu_{-n+1} \\
\vdots & \vdots & \ddots & \vdots \\
\mu_{n} & \mu_{n-1} & \cdots & \mu_{0}
\end{array}\right], \quad n \geq 0
$$

where $\mu_{n}=\int_{\mathcal{C}} \zeta^{-n} d \psi(\zeta)$, for $n=0, \pm 1, \pm 2, \ldots$, are the moments associated with the measure $\psi$. For example, $\mu_{0} \prod_{k=0}^{n-1}(1-$ $\left.\left|\alpha_{k}\right|^{2}\right)=\operatorname{det}\left(\mathbf{T}_{n}\right) / \operatorname{det}\left(\mathbf{T}_{n-1}\right), n \geq 1$.

For an easy understanding of this manuscript, we also present the definition of positive chain sequences (see Chihara's book [4]) which are used throughout this work. A sequence $\left\{a_{n}\right\}_{n=1}^{\infty}$ is a positive chain sequence if there exists a second sequence $\left\{g_{n}\right\}_{n=0}^{\infty}$ such that

$$
0 \leq g_{0}<1, \quad 0<g_{n}<1, \text { for } n \geq 1, \quad \text { and } a_{n}=\left(1-g_{n-1}\right) g_{n}, \text { for } n \geq 1 .
$$

The sequence $\left\{g_{n}\right\}_{n=0}^{\infty}$ is called a parameter sequence of the positive chain sequence $\left\{a_{n}\right\}_{n=1}^{\infty}$. The parameter sequence of a positive chain sequence can be non-unique. Every positive chain sequence has a minimal parameter sequence denoted by $\left\{m_{n}\right\}_{n=0}^{\infty}$, obtained with $m_{0}=0$. Every positive chain sequence has a maximal parameter sequence denoted by $\left\{M_{n}\right\}_{n=0}^{\infty}$, which is characterized by the condition that if $g_{0}>M_{0}$ then $\left\{g_{n}\right\}_{n=1}^{\infty}$ generated by $g_{n}=a_{n} /\left(1-g_{n-1}\right), n \geq 1$ does not satisfy $0<g_{n}<1, n \geq 1$. If $M_{0}=0$ then the minimal parameter sequence and the maximal parameter sequence coincide and we say that $\left\{a_{n}\right\}_{n=1}^{\infty}$ is a single parameter positive chain sequence (SPPCS).

Throughout this manuscript, notations of the form $S_{n}(\psi ; z)$ and $\alpha_{n}(\psi)=-\overline{S_{n+1}(\psi ; 0)}$ will be used to refer that these are, respectively, the monic OPUC and Verblunsky coefficients associated with the measure $\psi$.

The Lebesgue measure $\psi_{0}$ as a probability measure is given by $d \psi_{0}(z)=(2 \pi i z)^{-1} d z$ and the associated monic OPUC $S_{n}\left(\psi_{0}\right.$; $z)$ are simply

$$
S_{n}\left(\psi_{0} ; z\right)=z^{n}, \quad n \geq 0 .
$$

Considered as one of the examples in [8], Simon refers to this as the free case.

The aim of the present manuscript is to provide some explicit formulas regarding the OPUC, POPUC and Toeplitz matrices associated with measures which are simple modifications of the Lebesgue measure $\psi_{0}$.

The manuscript is organized as follows. In Section 2 we present a summary of all required theoretical results which were mainly obtained from [1] and [5]. Section 3 deals with the Verblunsky coefficients and the Toeplitz matrices related to the nontrivial probability measures $\psi_{1}^{(\kappa)}$, where

$$
\int_{\mathcal{C}} f(\zeta) d \psi_{1}^{(\kappa)}(\zeta)=(1-\kappa) \int_{\mathcal{C}} f(\zeta) \frac{1}{2 \pi i \zeta} d \zeta+\kappa f(i)
$$

for $0 \leq \kappa<1$. In Theorems 5 and 6 of Section 4 explicit formulas for certain POPUC satisfying three term recurrence relations are established for the measure $\psi_{1}^{(\kappa)}$. Finally, Section 5 deals with the OPUC associated with the nontrivial probability measures $\psi_{1}^{(\kappa)}\left(\lambda ;\right.$.) and $\psi_{2}^{(\kappa)}$, respectively, given by

$$
\int_{\mathcal{C}} f(\zeta) d \psi_{1}^{(\kappa)}(\lambda ; \zeta)=(1-\lambda)(1-\kappa) \int_{\mathcal{C}} f(\zeta) \frac{1}{2 \pi i \zeta} d \zeta+(1-\lambda) \kappa f(i)+\lambda f(1)
$$

and

$$
\int_{\mathcal{C}} f(\zeta) d \psi_{2}^{(\kappa)}(\zeta)=(1-\kappa) \int_{\mathcal{C}} f(\zeta) \frac{(1-\zeta)(\zeta-1)}{4 \pi i \zeta^{2}} d \zeta+\kappa f(i)
$$

The corresponding Verblunsky coefficients $\alpha_{n}\left(\psi_{1}^{(\kappa)}(\lambda ;).\right)$ and $\alpha_{n}\left(\psi_{2}^{(\kappa)}\right)$ are explicitly derived in Theorems 7 and 8 , respectively.

\section{Some preliminary results}

The results obtained in this manuscript are based on those shown in [1] and [5] connecting OPUC with POPUC that satisfy three term recurrence formula. First we summarize the results established in [5].

Given a nontrivial probability measure $\psi$ on the unit circle, assuming that it has a mass $\epsilon(0 \leq \epsilon<1)$ at $z=1$, we denote by $\{\psi(\lambda ;):. 0 \leq \lambda<1\}$, the family of nontrivial probability measures on the unit circle, where

$$
\int_{\mathcal{C}} f(\zeta) d \psi(\lambda ; \zeta)=\frac{1-\lambda}{1-\epsilon} \int_{\mathcal{C}} f(\zeta) d \psi(\zeta)+\frac{\lambda-\epsilon}{1-\epsilon} f(1), \quad 0 \leq \lambda<1
$$


Notice that if $\lambda>0$, then the probability measure $\psi(\lambda$; .) has a pure point of size $\lambda$ at $z=1$. Clearly, the measure $\psi$ is the member of the family of measures $\{\psi(\lambda ;$. ): $0 \leq \lambda<1\}$ at which $\lambda=\epsilon$.

It is well known that the sequence of Verblunsky coefficients $\left\{\alpha_{n}(\psi(\lambda ; .))\right\}_{n=0}^{\infty}$ associated with the measure $\psi(\lambda$; .) varies with the value of $\lambda$. However, there are sequences of numbers related to the family $\{\psi(\lambda ;$.): $0 \leq \lambda<1\}$ which are independent of the value $\lambda$. For one of such sequences, which we denote by $\left\{\rho_{n}(\psi)\right\}$, the following results hold.

$$
\begin{aligned}
& \rho_{0}(\psi)=\frac{S_{0}(\psi(\lambda ; .) ; 1)}{S_{0}^{*}(\psi(\lambda ; .) ; 1)}=1 \text { and } \\
& \rho_{n}(\psi)=\frac{S_{n}(\psi(\lambda ; .) ; 1)}{S_{n}^{*}(\psi(\lambda ; .) ; 1)}=\frac{\rho_{n-1}(\psi)-\overline{\alpha_{n-1}(\psi(\lambda ; .))}}{1-\rho_{n-1}(\psi) \alpha_{n-1}(\psi(\lambda ; .))}, \quad n \geq 1 .
\end{aligned}
$$

There are two other sequences, $\left\{c_{n}\right\}_{n=1}^{\infty}$ and $\left\{d_{n+1}\right\}_{n=1}^{\infty}$, independent of $\lambda$, which can be used to characterize the family of measures $\{\psi(\lambda ;):. 0 \leq \lambda<1\}$ as stated by the following theorem.

Theorem 1 ([5]). The family of nontrivial probability measures $\{\psi(\lambda ;$. ): $0 \leq \lambda<1\}$ on the unit circle can be uniquely mapped to a pair of real sequences $\left\{\left\{c_{n}\right\}_{n=1}^{\infty},\left\{d_{n+1}\right\}_{n=1}^{\infty}\right\}$, where $\left\{d_{n+1}\right\}_{n=1}^{\infty}$ is a positive chain sequence with its maximal parameter sequence $\left\{M_{n+1}\right\}_{n=0}^{\infty}$ such that $M_{1}>0$, as follows.

(a) Given $\left\{\psi(\lambda ;\right.$; ): $0 \leq \lambda<1\}$, the pair of sequences $\left\{\left\{c_{n}\right\}_{n=1}^{\infty},\left\{d_{n+1}\right\}_{n=1}^{\infty}\right\}$, which is independent of $\lambda$, satisfies

$$
\begin{aligned}
& c_{n}=c_{n}(\psi)=\frac{-\mathcal{I} m\left[\rho_{n-1}(\psi) \alpha_{n-1}(\psi(\lambda ; .))\right]}{1-\mathcal{R e}\left[\rho_{n-1}(\psi) \alpha_{n-1}(\psi(\lambda ; .))\right]}, \\
& d_{n+1}=d_{n+1}(\psi)=\left(1-g_{n}(\lambda)\right) g_{n+1}(\lambda), \quad n \geq 1,
\end{aligned}
$$

where the parameter sequence $\left\{g_{n+1}(\lambda)\right\}_{n=0}^{\infty}$ of $\left\{d_{n+1}\right\}_{n=1}^{\infty}$ is given by

$$
g_{n+1}(\lambda)=\frac{1}{2} \frac{\left|1-\rho_{n}(\psi) \alpha_{n}(\psi(\lambda ; .))\right|^{2}}{1-\mathcal{R} e\left[\rho_{n}(\psi) \alpha_{n}(\psi(\lambda ; .))\right]}, \quad n \geq 0 .
$$

The maximal parameter sequence $\left\{M_{n+1}\right\}_{n=0}^{\infty}$ of $\left\{d_{n+1}\right\}_{n=1}^{\infty}$ is such that $M_{n+1}=g_{n+1}(0), n \geq 0$, and that $M_{1} \geq g_{1}(\lambda)>0$.

Moreover, if $\left\{d_{n}(\lambda)\right\}_{n=1}^{\infty}$ is the positive chain sequence, where $d_{1}(\lambda)=g_{1}(\lambda)$ and $d_{n+1}(\lambda)=d_{n+1}, n \geq 1$, then the minimal $\left\{m_{n}(\lambda)\right\}_{n=0}^{\infty}$ and the maximal $\left\{M_{n}(\lambda)\right\}_{n=0}^{\infty}$ parameter sequences of $\left\{d_{n}(\lambda)\right\}_{n=1}^{\infty}$ are given by

$$
m_{0}(\lambda)=0, \quad M_{0}(\lambda)=\lambda, \quad m_{n}(\lambda)=g_{n}(\lambda), \quad M_{n}(\lambda)=M_{n}, \quad n \geq 1 .
$$

(b) Conversely, given a pair of real sequences $\left\{\left\{c_{n}\right\}_{n=1}^{\infty},\left\{d_{n+1}\right\}_{n=1}^{\infty}\right\}$, where $\left\{d_{n+1}\right\}_{n=1}^{\infty}$ is a positive chain sequence with its maximal parameter sequence $\left\{M_{n+1}\right\}_{n=0}^{\infty}$ such that $M_{1}>0$, let $\left\{m_{n}(\lambda)\right\}_{n=0}^{\infty}$ be the minimal parameter sequence of the positive chain sequence $\left\{d_{n}(\lambda)\right\}_{n=1}^{\infty}$, with $d_{1}(\lambda)=(1-\lambda) M_{1}$ and $d_{n+1}(\lambda)=d_{n+1}, n \geq 1$. Let

$$
\alpha_{n-1}(\lambda)=\frac{1}{\rho_{n-1}} \frac{1-2 m_{n}(\lambda)-i c_{n}}{1-i c_{n}}, \quad n \geq 1,
$$

where $\rho_{0}=1$ and $\rho_{n}=\rho_{n-1}\left(1-i c_{n}\right) /\left(1+i c_{n}\right), n \geq 1$. Then the associated set of nontrivial probability measures $\{\psi(\lambda ;): 0$. $\leq \lambda<1\}$ is such that $\alpha_{n}(\lambda)=\alpha_{n}(\psi(\lambda ;)),. n \geq 0$, and $\rho_{n}=\rho_{n}(\psi), n \geq 0$.

Moreover, the maximal parameter sequence $\left\{M_{n}(\lambda)\right\}_{n=0}^{\infty}$ of $\left\{d_{n}(\lambda)\right\}_{n=1}^{\infty}$ satisfies $M_{0}(\lambda)=\lambda$.

From the above Theorem 1 , if $\left\{R_{n}\right\}_{n=0}^{\infty}$ is the sequence of polynomials defined by the three term recurrence formula

$$
R_{n+1}(z)=\left[\left(1+i c_{n+1}\right) z+\left(1-i c_{n+1}\right)\right] R_{n}(z)-4 d_{n+1} z R_{n-1}(z), \quad n \geq 1,
$$

with $R_{0}(z)=1$ and $R_{1}(z)=\left(1+i c_{1}\right) z+\left(1-i c_{1}\right)$, then $(z-1) R_{n}(z)$ are the (appropriately scaled) POPUC given by

$$
\begin{aligned}
& (z-1) R_{n}(z)=\frac{\prod_{j=0}^{n-1}\left[1-\rho_{j}(\psi) \alpha_{j}(\psi(\lambda ; .))\right]}{\prod_{j=0}^{n-1}\left[1-\mathcal{R e}\left[\rho_{j}(\psi) \alpha_{j}(\psi(\lambda ; .))\right]\right]}\left[z S_{n}(\psi(\lambda ; .) ; z)-\rho_{n}(\psi) S_{n}^{*}(\psi(\lambda ; .) ; z)\right], \\
& =\frac{\prod_{j=0}^{n-1}\left[1-\rho_{j}(\psi) \alpha_{j}(\psi)\right]}{\prod_{j=0}^{n-1}\left[1-\mathcal{R e}\left[\rho_{j}(\psi) \alpha_{j}(\psi)\right]\right]}\left[z S_{n}(\psi(\lambda ; .) ; z)-\rho_{n}(\psi) S_{n}^{*}(\psi(\lambda ; .) ; z)\right],
\end{aligned}
$$

for $n \geq 0$.

For any sequence of polynomials $\left\{R_{n}\right\}_{n=0}^{\infty}$ satisfying a three term recurrence formula of the type (6), see [1,3], one can also associate a moment functional $\mathcal{N}$ and the so called L-orthogonality property

$$
\mathcal{N}\left[z^{-n+j} R_{n}(z)\right]= \begin{cases}0, & 0 \leq j \leq n-1, \\ \gamma_{n}, & j=n\end{cases}
$$

where

$$
\gamma_{0}=\frac{2 d_{1}}{1+i c_{1}}, \quad \gamma_{n}=\frac{4 d_{n+1}}{1+i c_{n+1}} \gamma_{n-1}, \quad n \geq 1,
$$


and $d_{1} \neq 0$ is arbitrary. However, for the polynomials $R_{n}$ provided in $(7)$, we have $\mathcal{N}[\lambda ; \ell]=$ $\int_{\mathcal{C}} \ell(\zeta)(1-\zeta) d \psi(\lambda ; \zeta)$ and, hence, the L-orthogonality property

$$
\mathcal{N}\left[\lambda ; z^{-n+j} R_{n}(z)\right]=\int_{\mathcal{C}} \zeta^{-n+j} R_{n}(\zeta)(1-\zeta) d \psi(\lambda ; \zeta)= \begin{cases}0, & 0 \leq j \leq n-1, \\ \gamma_{n}(\lambda), & j=n,\end{cases}
$$

where

$$
\gamma_{0}(\lambda)=\frac{2 d_{1}(\lambda)}{1+i c_{1}} \quad \text { and } \quad \gamma_{n}(\lambda)=\frac{4 d_{n+1}}{1+i c_{n+1}} \gamma_{n-1}(\lambda), \quad n \geq 1
$$

As stated in [3], we can also write

$$
S_{n}(\psi(\lambda ; .) ; z)=\frac{1}{\prod_{k=1}^{n}\left(1+i c_{k}\right)}\left[R_{n}(z)-2\left(1-m_{n}(\lambda)\right) R_{n-1}(z)\right], \quad n \geq 1 .
$$

The polynomials $R_{n}$ are actually constant multiples of the $\operatorname{CD}$ kernels $K_{n}(\lambda ; z, 1)=$ $\sum_{j=0}^{n} \overline{s_{j}(\psi(\lambda ; .) ; 1)} s_{j}(\psi(\lambda ;) ; z$.$) associated with the probability measure \psi(\lambda ;$.$) . Here, s_{n}(\psi(\lambda ;) ; z$.$) are the orthonormal$ polynomials associated with $\psi(\lambda$; .). For other studies that use the connection between CD kernels and para-orthogonal polynomials we refer to Cantero et al. [2] and Golinskii [6]. For a much more recent paper exploring this connection see [13]. We also cite [10], which provides a nice section on para-orthogonal polynomials.

There is another technique to map a nontrivial probability measure $\psi$ to some pair of real sequences $\left\{\left\{c_{n}\right\}_{n=1}^{\infty},\left\{d_{n+1}\right\}_{n=1}^{\infty}\right\}$, where $\left\{d_{n+1}\right\}_{n=1}^{\infty}$ is a positive chain sequence. The following theorem establishes such technique and it is obtained from results contained in [1].

\section{Theorem 2 ([1]).}

(a) Given the nontrivial probability measure $\phi$ on the unit circle and the number $\tau$ such that $|\tau|=1$ and $\tau \neq 1$, then associated with the pair $(\phi, \tau)$ there exists a unique pair of real sequences $\left\{\left\{c_{n}\right\}_{n=1}^{\infty},\left\{d_{n+1}\right\}_{n=1}^{\infty}\right\}$, where $\left\{d_{n+1}\right\}_{n=1}^{\infty}$ is also a positive chain sequence and

$$
\begin{aligned}
& c_{1}=c_{1}(\phi, \tau)=i \frac{\rho_{0}(\phi, \tau)+1}{\rho_{0}(\phi, \tau)-1} \\
& c_{n+1}=c_{n+1}(\phi, \tau)=\frac{-\mathcal{I} m\left[\rho_{n}(\phi, \tau) \alpha_{n-1}(\phi)\right]}{1+\mathcal{R} e\left[\rho_{n}(\phi, \tau) \alpha_{n-1}(\phi)\right]}, \\
& d_{n+1}=d_{n+1}(\phi, \tau)=\left[1-\mathfrak{m}_{n-1}(\phi, \tau)\right] \mathfrak{m}_{n}(\phi, \tau), \quad n \geq 1 .
\end{aligned}
$$

Here, $\left\{\alpha_{n}(\phi)\right\}_{n=0}^{\infty}$ is the sequence of Verblunsky coefficients associated with $\phi$, and the sequences $\left\{\rho_{n}(\phi, \tau)\right\}_{n=0}^{\infty}$ and $\left\{\mathfrak{m}_{n}(\phi, \tau)\right\}_{n=0}^{\infty}$ satisfy

$$
\begin{aligned}
& \rho_{0}(\phi, \tau)=\tau, \quad \rho_{n}(\phi, \tau)=\frac{\rho_{n-1}(\phi, \tau)-\overline{\alpha_{n-1}(\phi)}}{1-\rho_{n-1}(\phi, \tau) \alpha_{n-1}(\phi)}, \quad n \geq 1 \\
& \mathfrak{m}_{0}(\phi, \tau)=0 \quad \text { and } \quad \mathfrak{m}_{n}(\phi, \tau)=\frac{1}{2} \frac{1-\left|\rho_{n}(\phi, \tau) \alpha_{n-1}(\phi)\right|^{2}}{1+\mathcal{R e}\left[\rho_{n}(\phi, \tau) \alpha_{n-1}(\phi)\right]}, \quad n \geq 1 .
\end{aligned}
$$

(b) Conversely, given the pair of real sequences $\left\{\left\{c_{n}\right\}_{n=1}^{\infty},\left\{d_{n+1}\right\}_{n=1}^{\infty}\right\}$, where $\left\{d_{n+1}\right\}_{n=1}^{\infty}$ is also a positive chain sequence, let $\tau$, $\left\{\rho_{n}\right\}_{n=0}^{\infty}$ and $\left\{\alpha_{n}\right\}_{n=0}^{\infty}$ be such that

$$
\begin{aligned}
& \tau=-\frac{1-i c_{1}}{1+i c_{1}} \\
& \rho_{0}=\tau, \quad \rho_{n}=\frac{1-i c_{n+1}}{1+i c_{n+1}} \rho_{n-1}, \quad n \geq 1,
\end{aligned}
$$

and

$$
\alpha_{n-1}=\frac{1}{\rho_{n}} \frac{1-2 \mathfrak{m}_{n}-i c_{n+1}}{1+i c_{n+1}}, \quad n \geq 1 .
$$

Here, $\left\{\mathfrak{m}_{n}\right\}_{n=0}^{\infty}$, is the minimal parameter sequence of $\left\{d_{n+1}\right\}_{n=1}^{\infty}$. Then associated with $\left\{\left\{c_{n}\right\}_{n=1}^{\infty},\left\{d_{n+1}\right\}_{n=1}^{\infty}\right\}$ there exists a unique nontrivial probability measure on the unit circle $\phi$ such that $\alpha_{n-1}=\alpha_{n-1}(\phi), c_{n}=c_{n}(\phi, \tau)$ and $d_{n+1}=d_{n+1}(\phi, \tau), n \geq 1$.

As shown in [1], the results in the above theorem are based on the following facts.

If $\left\{R_{n}(\tau ; z)\right\}_{n=0}^{\infty}$ is the sequence of polynomials obtained by the three term recurrence formula

$$
R_{n+1}(\tau ; z)=\left[\left(1+i c_{n+1}(\phi, \tau)\right) z+\left(1-i c_{n+1}(\phi, \tau)\right)\right] R_{n}(\tau ; z)-4 d_{n+1}(\phi, \tau) z R_{n-1}(\tau ; z), \quad n \geq 1,
$$

with $R_{0}(\tau ; z)=1$ and $R_{1}(\tau ; z)=\left(1+i c_{1}(\phi, \tau)\right) z+\left(1-i c_{1}(\phi, \tau)\right)$, then $R_{n}(\tau ; z)$ are the (appropriately scaled) POPUC given by

$$
R_{n}(\tau ; z)=\prod_{k=0}^{n-1} \frac{1+\overline{\rho_{k}(\phi, \tau) \alpha_{k-1}(\phi)}}{1+\mathcal{R e}\left[\rho_{k}(\phi, \tau) \alpha_{k-1}(\phi)\right]}\left[z S_{n-1}(\phi ; z)-\rho_{n-1}(\phi, \tau) S_{n-1}^{*}(\phi ; z)\right], \quad n \geq 1
$$


Reciprocally, we can also write

$$
(z-1) S_{n}(\phi ; z)=\frac{1}{\prod_{k=1}^{n+1}\left[1+i c_{k}(\phi, \tau)\right]}\left[R_{n+1}(\tau ; z)-2\left[1-\mathfrak{m}_{n}(\phi, \tau)\right] R_{n}(\tau ; z)\right], \quad n \geq 0 .
$$

Moreover, if the measure $\phi$ is such that the principal value integral $I=f_{\mathcal{C}} \zeta(\zeta-1)^{-1} d \phi(\zeta)$ exists, then we can give the following information about the moment functional $\mathcal{N}=\mathcal{N}^{(\tau(t))}$ associated with the three term recurrence formula (9), with an additional element $d_{1} \neq 0$.

In Theorem 2, if $\tau$ satisfies

$$
\tau=\tau(t)=-\frac{I+i t}{\bar{I}-i t},
$$

then

$$
\mathcal{N}^{(\tau(t))}[\ell]=\frac{4 d_{1}}{1+4(t+\mathcal{I} m(I))^{2}}\left[f_{\mathcal{C}} \ell(\zeta) \frac{\zeta}{\zeta-1} d \phi(\zeta)+i t \ell(1)\right]
$$

for any Laurent polynomial $\ell$ and

$$
\mathcal{N}^{(\tau(t))}\left[z^{-n+j} R_{n}(\tau(t) ; z)\right]= \begin{cases}0, & 0 \leq j \leq n-1, \\ \gamma_{n}(\tau(t)), & j=n,\end{cases}
$$

where

$$
\gamma_{0}(\tau(t))=\frac{2 d_{1}}{1+i c_{1}(\phi, \tau(t))} \quad \text { and } \quad \gamma_{n}(\tau(t))=\frac{4 d_{n+1}(\phi, \tau(t))}{1+i c_{n+1}(\phi, \tau(t))} \gamma_{n-1}(\tau(t)), \quad n \geq 1 .
$$

Clearly, the moment functional $\mathcal{N}^{(\tau(t))}$ satisfies $\mathcal{N}^{(\tau(t))}[1]=\frac{4 d_{1}}{1+4(t+\mathcal{I} m(I))^{2}}[I+i t]$ and

$$
\begin{aligned}
& \mathcal{N}^{(\tau(t))}\left[z^{n}\right]=\frac{4 d_{1}}{1+4(t+\mathcal{I} m(I))^{2}}\left[I+\sum_{j=1}^{n} \int_{\mathcal{C}} \zeta^{j} d \phi(\zeta)+i t\right], \\
& \mathcal{N}^{(\tau(t))}\left[z^{-n}\right]=\frac{4 d_{1}}{1+4(t+\mathcal{I} m(I))^{2}}\left[I-\sum_{j=1}^{n} \int_{\mathcal{C}} \zeta^{-n+j} d \phi(\zeta)+i t\right], \quad n \geq 1 .
\end{aligned}
$$

We can also say more about the positive chain sequences $\left\{d_{n+1}(\phi, \tau(t))\right\}_{n=1}^{\infty}$.

If $t \neq 0$ then $\left\{d_{n+1}(\phi, \tau(t))\right\}_{n=1}^{\infty}$ is always a SPPCS. However, $\left\{d_{n+1}(\tau(0))\right\}_{n=1}^{\infty}$ is not a SPPCS if and only if the measure $\phi$ is such that the integral $J=\int_{\mathcal{C}} \zeta(1-\zeta)^{-1}(\zeta-1)^{-1} d \phi(\zeta)$ exists.

When $J$ exists, by considering $R_{n}(\tau(0) ; z), n \geq 0$, to be the same polynomials given immediately after Theorem 1 , we have for any Laurent polynomial $\ell$,

$$
\mathcal{N}[\lambda ; \ell]=\int_{\mathcal{C}} \ell(\zeta)(1-\zeta) d \psi(\lambda ; \zeta)=\frac{4 d_{1}(\lambda)}{1+(2 \mathcal{I} m(I))^{2}} \int_{\mathcal{C}} \ell(\zeta) \frac{\zeta}{\zeta-1} d \phi(\zeta)=\mathcal{N}^{(\tau(0))}[\ell]
$$

where $d_{1}$ in $\mathcal{N}^{(\tau(0))}$ is assigned the value $d_{1}(\lambda)=(1-\lambda) M_{1}$ as in Theorem 1. In conclusion, as also shown in [1], we can state the following.

Theorem 3 ([1]]). Given the pair of real sequences $\left\{\left\{c_{n}\right\}_{n=1}^{\infty},\left\{d_{n+1}\right\}_{n=1}^{\infty}\right\}$, where $\left\{d_{n+1}\right\}_{n=1}^{\infty}$ is a positive chain sequence with its maximal parameter sequence $\left\{M_{n+1}\right\}_{n=0}^{\infty}$ such that $M_{1}>0$ (i.e., not a SPPCS). Let $\psi(\lambda ;$. ) and $\phi$ be the nontrivial probability measures obtained, respectively, in the part $b$ ) of Theorem 1 and in part $b)$ of Theorem 2 . Then

$$
\frac{4 d_{1}(\lambda)}{1+(2 \mathcal{I} m(I))^{2}} d \phi(z)=\frac{(z-1)(1-z)}{z} d \psi(\lambda ; z) .
$$

Here, $I=\int_{\mathcal{C}} \zeta(\zeta-1)^{-1} d \phi(\zeta)$.

\section{OPUC associated with $\psi_{1}^{(\kappa)}$}

To be able to obtain information about the OPUC associated with any of the measures $\psi_{1}^{(\kappa)}, 0 \leq \kappa<1$, we start with the family of nontrivial probability measures $\left\{\psi_{0}(\lambda ;):. 0 \leq \lambda<1\right\}$, where $\psi_{0}(z)=\psi_{0}(0 ; z)$ is the Lebesgue measure stated in the Introduction. It is well known that the sequence of Verblunsky coefficients associated with $\psi_{0}\left(\lambda ;\right.$; ) is the null sequence $\{0\}_{n=0}^{\infty}$ and, hence, the sequences of numbers $\left\{\rho_{n}\right\}_{n=0}^{\infty},\left\{c_{n}\right\}_{n=1}^{\infty}$ and $\left\{d_{n+1}\right\}_{n=1}^{\infty}$ that follow from Theorem 1 , for $\lambda=0$, are

$$
\rho_{n-1}=1, \quad c_{n}=0 \quad \text { and } \quad d_{n+1}=\left(1-g_{n}(0)\right) g_{n+1}(0)=\frac{1}{4}, \quad n \geq 1 .
$$

Observe also that the parameter sequence $\left\{g_{n+1}(0)\right\}_{n=0}^{\infty}$ obtained for the above $\left\{d_{n+1}\right\}_{n=1}^{\infty}$ is the maximal parameter sequence $\left\{M_{n+1}\right\}_{n=0}^{\infty}$ such that $M_{n+1}=1 / 2, n \geq 0$. 
The para-orthogonal polynomials given by (7) are

$$
(z-1) R_{n}(z)=z^{n+1}-1, \quad n \geq 0 .
$$

Now if we consider the positive chain sequence $\left\{d_{n}(\lambda)\right\}_{n=1}^{\infty}$, where $d_{1}(\lambda)=(1-\lambda) M_{1}=(1-\lambda) / 2$ and $d_{n+1}(\lambda)=d_{n+1}=1 / 4$, $n \geq 1$, then one can easily verify that the minimal parameter sequence $\left\{m_{n}(\lambda)\right\}_{n=0}^{\infty}$ of $\left\{d_{n}(\lambda)\right\}_{n=1}^{\infty}$ satisfies

$$
m_{0}(\lambda)=0, \quad m_{n}(\lambda)=\frac{1}{2} \frac{1+(n-2) \lambda}{1+(n-1) \lambda}, \quad n \geq 1 .
$$

Hence, from Theorem 1 , the Verblunsky coefficients $\alpha_{n}\left(\psi_{0}(\lambda ;).\right)$ associated with $\psi_{0}(\lambda ;$.) are

$$
\alpha_{n}\left(\psi_{0}(\lambda ; .)\right)=\frac{\lambda}{1+n \lambda}, \quad n \geq 0
$$

To obtain the Verblunsky coefficients $\left\{\alpha_{n}\left(\psi_{1}^{(\kappa)}\right)\right\}_{n=0}^{\infty}$ associated with the measure $\psi_{1}^{(\kappa)}$, we first observe that

$$
d \psi_{1}^{(\kappa)}(z)=d \psi_{0}\left(\kappa ; e^{-i \pi / 2} z\right)
$$

Since it is known that $\alpha_{n}(\omega \psi)=(\omega)^{n+1} \alpha_{n}(\psi)$, for $|\omega|=1$, using $\omega=e^{-i \pi / 2}$, we immediately get, for the Verblunsky coefficients of the measure $\psi_{1}^{(\kappa)}$, that $\alpha_{n}\left(\psi_{1}^{(\kappa)}\right)=\left(e^{-i \pi / 2}\right)^{n+1} \alpha_{n}\left(\psi_{0}(\kappa ;).\right)$ and thus

$$
\alpha_{n}\left(\psi_{1}^{(\kappa)}\right)=\frac{(-i)^{n+1} \kappa}{1+n \kappa}, \quad n \geq 0
$$

Now we give some information about the Toeplitz matrices associated with the measure $\psi_{1}^{(\kappa)}$, where the moments $\mu_{n}=$ $\int_{0}^{2 \pi} e^{-i n \theta} d \psi_{1}^{(\kappa)}\left(e^{i \theta}\right), n=0, \pm 1, \pm 2, \ldots$, are easily found to be

$$
\mu_{0}=1 \text { and } \mu_{n}=(i)^{-n} \kappa, \quad n= \pm 1, \pm 2, \ldots
$$

Theorem 4. The $(n+1) \times(n+1)$ Toeplitz matrix $\mathbf{T}_{n}$ associated with the measure $\psi_{1}^{(\kappa)}, 0 \leq \kappa<1$, defined in (2) has the $\mathbf{L}_{n} \mathbf{D}_{n} \mathbf{L}_{n}^{H}$ factorization, where

$$
\mathbf{D}_{n}=\left[\begin{array}{cccc}
\mathfrak{d}_{1,1} & 0 & \cdots & 0 \\
0 & \mathfrak{d}_{2,2} & \cdots & 0 \\
\vdots & \vdots & \ddots & \vdots \\
0 & 0 & \cdots & \mathfrak{d}_{n+1, n+1}
\end{array}\right], \quad \mathbf{L}_{n}=\left[\begin{array}{cccc}
1 & 0 & \cdots & 0 \\
\ell_{2,1} & 1 & \cdots & 0 \\
\vdots & \vdots & \ddots & \vdots \\
\ell_{n+1,1} & \ell_{n+1,2} & \cdots & 1
\end{array}\right], \quad n \geq 1,
$$

are such that

$$
\mathfrak{d}_{k k}=\frac{1+(k-1) \kappa}{1+(k-2) \kappa}(1-\kappa), \quad k=1,2, \ldots, n+1,
$$

and

$$
\ell_{j, k}=\frac{(i)^{k-j} \kappa}{1+(k-1) \kappa}, \quad j=k+1, \ldots, n+1, \quad k=1, \ldots, n .
$$

Proof. We consider the $\mathbf{L}_{n} \mathbf{U}_{n}$ factorization, where

$$
\mathbf{U}_{n}=\left[\begin{array}{cccc}
u_{1,1} & u_{1,2} & \cdots & u_{1, n+1} \\
0 & u_{2,2} & \cdots & u_{2, n+1} \\
\vdots & \vdots & \ddots & \vdots \\
0 & 0 & \cdots & u_{n+1, n+1}
\end{array}\right]
$$

and show that, in addition to the above values for $\ell_{k, j}$ that

$$
u_{k, k}=\frac{1+(k-1) \kappa}{1+(k-2) \kappa}(1-\kappa), \quad u_{k, j}=\frac{(i)^{j-k} \kappa(1-\kappa)}{1+(k-2) \kappa}, j=k+1, k+2, \ldots, n+1,
$$

for $k=1,2, \ldots, n+1$.

From the Doolittle algorithm for such LU factorization (see for example [12, p. 53]), we can successively obtain the values of $u_{k, j}$ and $\ell_{j, k}$ as follows

$$
u_{1,1}=\mu_{0}=1, \quad u_{1, j}=\mu_{1-j}=(i)^{j-1} \kappa, j=2,3, \ldots, n+1,
$$

and

$$
\ell_{j, 1}=\frac{\mu_{j-1}}{u_{1,1}}=(i)^{1-j} \kappa, \quad j=2,3, \ldots, n+1
$$

Hence, the expressions for $u_{k, j}$ and $\ell_{j, k}$ are true for $k=1$. 
Now suppose that the expressions for $u_{k, j}$ and $\ell_{j, k}$ are true for the values of $k=1,2, \ldots, m-1$ and we show that it is also true for $k=m$. First we have from the expression for $u_{m, j}$ in the Doolittle algorithm

$$
u_{m, j}=\mu_{m-j}-\sum_{s=1}^{m-1} \ell_{m, s} u_{s, j}=\mu_{m-j}-\sum_{s=1}^{m-1} \frac{(i)^{s-m} \kappa}{1+(s-1) \kappa} \frac{(i)^{j-s} \kappa(1-\kappa)}{1+(s-2) \kappa},
$$

for $j=m, m+1, \ldots, n+1$. Considering the products within the above sum, since

$$
\frac{1-\kappa}{[1+(s-1) \kappa][1+(s-2) \kappa]}=\frac{s}{1+(s-1) \kappa}-\frac{s-1}{1+(s-2) \kappa},
$$

we conclude immediately that

$$
u_{m, m}=\frac{1+(m-1) \kappa}{1+(m-2) \kappa}(1-\kappa), \quad u_{m, j}=\frac{(i)^{j-m} \kappa(1-\kappa)}{1+(m-2) \kappa}, j=m+1, \ldots, n+1 .
$$

From the expression for $\ell_{j, m}$ in the Doolittle algorithm, we have

$$
\ell_{j, m} u_{m, m}=\mu_{j-m}-\sum_{s=1}^{m-1} \ell_{j, s} u_{s, m}=\mu_{j-m}-\sum_{s=1}^{m-1} \frac{(i)^{s-j} \kappa}{1+(s-1) \kappa} \frac{(i)^{m-s} \kappa(1-\kappa)}{1+(s-2) \kappa},
$$

for $j=m+1, \ldots, n+1$. Again using (12), we easily obtain that

$$
\ell_{j, m}=\frac{(i)^{m-j} \kappa}{1+(m-1) \kappa}, \quad j=m+1, \ldots, n+1 .
$$

Hence, we conclude the results for $\mathbf{L}_{n}$ and $\mathbf{U}_{n}$ by induction. Finally, since the matrix $\mathbf{T}_{n}$ is Hermitian, it is easily verified that $\mathbf{U}_{n}=\mathbf{D}_{n} \mathbf{L}_{n}^{H}$.

\section{POPUC associated with $\psi_{1}^{(\kappa)}$}

For each $\kappa, 0 \leq \kappa<1$, we can also define the family of probability measures $\left\{\psi_{1}^{(\kappa)}(\lambda ;):. 0 \leq \lambda<1\right\}$ using (4). Observing that $\psi_{1}^{(\kappa)}(z)=\psi_{1}^{(\kappa)}(0 ; z)$, we have $\left\{\psi_{1}^{(\kappa)}(\lambda ;):. 0 \leq \lambda<1\right\}$ such that

$$
\begin{aligned}
\int_{\mathcal{C}} f(\zeta) d \psi_{1}^{(\kappa)}(\lambda ; \zeta) & =(1-\lambda) \int_{\mathcal{C}} f(\zeta) d \psi_{1}^{(\kappa)}(\zeta)+\lambda f(1) \\
& =(1-\lambda)(1-\kappa) \int_{\mathcal{C}} f(\zeta) \frac{1}{2 \pi i \zeta} d \zeta+(1-\lambda) \kappa f(i)+\lambda f(1) .
\end{aligned}
$$

Recalling what was stated in the beginning of Section 2 , the Verblunsky coefficients $\alpha_{n}\left(\psi_{1}^{(\kappa)}(\lambda ;).\right)$ associated with the measure $\psi_{1}^{(\kappa)}\left(\lambda ;\right.$.) vary with the values of $\kappa$ and $\lambda$. However, the coefficients $\rho_{n}\left(\psi_{1}^{(\kappa)}\right)=S_{n}\left(\psi_{1}^{(\kappa)}(\lambda ;) ; 1.\right) / S_{n}^{*}\left(\psi_{1}^{(\kappa)}(\lambda ;) ; 1.\right), n \geq 1$, are independent of $\lambda$. From (5)

$$
\rho_{0}\left(\psi_{1}^{(\kappa)}\right)=1, \quad \rho_{n}\left(\psi_{1}^{(\kappa)}\right)=\frac{\rho_{n-1}\left(\psi_{1}^{(\kappa)}\right)-\overline{\alpha_{n-1}\left(\psi_{1}^{(\kappa)}(0 ; .)\right)}}{1-\rho_{n-1}\left(\psi_{1}^{(\kappa)}\right) \alpha_{n-1}\left(\psi_{1}^{(\kappa)}(0 ; .)\right)}, \quad n \geq 1,
$$

and hence, from $\psi_{1}^{(\kappa)}(z)=\psi_{1}^{(\kappa)}(0 ; z)$ and (11), we easily verify by induction that

$$
\begin{aligned}
& \rho_{4 m}\left(\psi_{1}^{(\kappa)}\right)=1, \quad \rho_{4 m+1}\left(\psi_{1}^{(\kappa)}\right)=\frac{4 m \kappa+1-i \kappa}{4 m \kappa+1+i \kappa}, \\
& \rho_{4 m+2}\left(\psi_{1}^{(\kappa)}\right)=\frac{(4 m+2) \kappa+1-i \kappa}{(4 m+2) \kappa+1+i \kappa}, \quad \rho_{4 m+3}\left(\psi_{1}^{(\kappa)}\right)=1, \quad m \geq 0 .
\end{aligned}
$$

Hence, as in (7), considering the polynomials

$$
R_{n}^{(\kappa)}(z)=\frac{\prod_{j=0}^{n-1}\left[1-\rho_{j}\left(\psi_{1}^{(\kappa)}\right) \alpha_{j}\left(\psi_{1}^{(\kappa)}\right)\right]}{\prod_{j=0}^{n-1}\left[1-\mathcal{R} e\left[\rho_{j}\left(\psi_{1}^{(\kappa)}\right) \alpha_{j}\left(\psi_{1}^{(\kappa)}\right)\right]\right]} \frac{z S_{n}\left(\psi^{(\kappa)}(\lambda ; .) ; z\right)-\rho_{n}\left(\psi_{1}^{(\kappa)}\right) S_{n}^{*}\left(\psi^{(\kappa)}(\lambda ; .) ; z\right)}{z-1},
$$

which are independent of $\lambda$, we obtain from Theorem 1 the following.

Theorem 5. For the sequence of polynomials $\left\{R_{n}^{(\kappa)}\right\}_{n=0}^{\infty}$ given above the following hold.

$$
R_{n+1}^{(\kappa)}(z)=\left[\left(1+i c_{n+1}^{(\kappa)}\right) z+\left(1-i c_{n+1}^{(\kappa)}\right)\right] R_{n}^{(\kappa)}(z)-4 d_{n+1}^{(\kappa)} z R_{n-1}^{(\kappa)}(z),
$$

with $R_{0}^{(\kappa)}(z)=1$ and $R_{1}^{(\kappa)}(z)=\left(1+i c_{1}^{(\kappa)}\right) z+\left(1-i c_{1}^{(\kappa)}\right)$, and

$$
\mathcal{N}^{(\kappa)}\left[\lambda ; z^{-n+j} R_{n}(z)\right]=\int_{\mathcal{C}} \zeta^{-n+j} R_{n}(\zeta)(1-\zeta) d \psi_{1}^{(\kappa)}(\lambda ; \zeta)= \begin{cases}0, & 0 \leq j \leq n-1 \\ \gamma_{n}^{(\kappa)}(\lambda), & j=n\end{cases}
$$


where

$$
\gamma_{0}^{(\kappa)}(\lambda)=\frac{2(1-\lambda) M_{1}^{(\kappa)}}{1+i c_{1}^{(\kappa)}} \text { and } \quad \gamma_{n}^{(\kappa)}(\lambda)=\frac{4 d_{n+1}^{(\kappa)}}{1+i c_{n+1}^{(\kappa)}} \gamma_{n-1}^{(\kappa)}(\lambda), \quad n \geq 1 .
$$

Here, $\left\{c_{n}^{(\kappa)}\right\}_{n=1}^{\infty}$ and the maximal parameter sequence $\left\{M_{n+1}^{(\kappa)}\right\}_{n=0}^{\infty}$ of the positive chain sequence $\left\{d_{n+1}^{(\kappa)}\right\}_{n=1}^{\infty}$ are explicitly given by

$$
\begin{aligned}
c_{4 m+1}^{(\kappa)} & =\frac{\kappa}{4 m \kappa+1}, \quad M_{4 m+1}^{(\kappa)}=\frac{1}{2} \frac{(4 m \kappa+1)^{2}+\kappa^{2}}{(4 m \kappa+1)^{2}}, \\
c_{4 m+2}^{(\kappa)} & =\frac{-2 \kappa^{2}}{[(4 m+1) \kappa+1]^{2}}, \quad M_{4 m+2}^{(\kappa)}=\frac{1}{2} \frac{(4 m \kappa+1)\left[[(4 m+2) \kappa+1]^{2}+\kappa^{2}\right]}{[(4 m+1) \kappa+1]^{3}}, \\
c_{4 m+3}^{(\kappa)} & =\frac{-\kappa}{(4 m+2) \kappa+1}, \quad M_{4 m+3}^{(\kappa)}=\frac{1}{2} \frac{[(4 m+2) \kappa+1]^{2}-\kappa^{2}}{[(4 m+2) \kappa+1]^{2}}, \\
c_{4 m+4}^{(\kappa)} & =0, \quad M_{4 m+4}^{(\kappa)}=\frac{1}{2} \frac{(4 m+2) \kappa+1}{(4 m+3) \kappa+1},
\end{aligned}
$$

for $m \geq 0$.

Proof. The results for $\left\{c_{n}^{(\kappa)}\right\}_{n=1}^{\infty}$ and $\left\{M_{n+1}^{(\kappa)}\right\}_{n=0}^{\infty}=\left\{g_{n+1}^{(\kappa)}(0)\right\}_{n=1}^{\infty}$ are straightforward substitutions of the respective values of $\rho_{n}\left(\psi_{1}^{(\kappa)}\right)$ obtained in (14) and $\alpha_{n}\left(\psi_{1}^{(\kappa)}(0 ;).\right)=\alpha_{n}\left(\psi_{1}^{(\kappa)}\right)$ given by (11) in the results of part a) of Theorem 1.

Observe that, as stated in part b) of Theorem 1 ,

$$
\prod_{k=1}^{n} \frac{1-i c_{k}^{(\kappa)}}{1+i c_{k}^{(\kappa)}}=\rho_{n}\left(\psi_{1}^{(\kappa)}\right), \quad n \geq 1
$$

where $\rho_{n}\left(\psi_{1}^{(\kappa)}\right)$ are given explicitly in (14).

The measure $\psi_{1}^{(\kappa)}$ is such that the integral $J=\int_{\mathcal{C}} \zeta(1-\zeta)^{-1}(\zeta-1)^{-1} d \psi_{1}^{(\kappa)}(\zeta)$ does not exist. However, we can verify that the principal value integral $I=f_{\mathcal{C}} \zeta(\zeta-1)^{-1} d \psi_{1}^{(\kappa)}(\zeta)$ exists as follows.

$$
I=\int_{\mathcal{C}} \frac{\zeta}{\zeta-1} d \psi_{1}^{(\kappa)}(\zeta)=(1-\kappa) \lim _{\epsilon \rightarrow 0} \frac{1}{2 \pi} \int_{0+\epsilon}^{2 \pi-\epsilon} \frac{e^{i \theta}}{e^{i \theta}-1} d \theta+\kappa \frac{i}{i-1} .
$$

This can be written as

$$
I-\kappa \frac{i}{i-1}=(1-\kappa) \frac{1}{2 \pi} \lim _{\epsilon \rightarrow 0}\left[\int_{0+\epsilon}^{\pi} \frac{e^{i \theta}}{e^{i \theta}-1} d \theta+\int_{0+\epsilon}^{\pi} \frac{e^{-i \theta}}{e^{-i \theta}-1} d \theta\right]=(1-\kappa) \frac{1}{2 \pi} \lim _{\epsilon \rightarrow 0} \int_{0+\epsilon}^{\pi} d \theta,
$$

from which $I=(1-i \kappa) / 2$.

Hence, we can use Theorem 2 with

$$
\tau=\tau(t)=-\frac{I+i t}{\bar{I}-i t}=-\frac{1-i(\kappa-2 t)}{1+i(\kappa-2 t)},
$$

to obtain POPUC, say $R_{n}(\tau(t) ; z)=R_{n}\left(\psi_{1}^{(\kappa)}, t ; z\right)$, which satisfy three term recurrence formulas and L-orthogonal property. From Theorem 2 we obtain, for the numbers $\rho_{n}\left(\psi_{1}^{(\kappa)}, t\right)=\rho_{n}\left(\psi_{1}^{(\kappa)}, \tau(t)\right), c_{n}\left(\psi_{1}^{(\kappa)}, t\right)=c_{n}\left(\psi_{1}^{(\kappa)}, \tau(t)\right)$ and $\mathfrak{m}_{n}\left(\psi_{1}^{(\kappa)}, t\right)=$ $\mathfrak{m}_{n}\left(\psi_{1}^{(\kappa)}, \tau(t)\right)$, that

$$
\begin{aligned}
\rho_{4 m}\left(\psi_{1}^{(\kappa)}, t\right) & =-\frac{(1-\kappa)[1+(4 m-i) \kappa]+i 2 t[1+(4 m-1) \kappa]}{(1-\kappa)[1+(4 m+i) \kappa]-i 2 t[1+(4 m-1) \kappa]}, \\
\rho_{4 m+1}\left(\psi_{1}^{(\kappa)}, t\right) & =-\frac{(1-\kappa)[1+(4 m+1) \kappa]+2 t \kappa+i 2 t[1+(4 m) \kappa]}{(1-\kappa)[1+(4 m+1) \kappa]+2 t \kappa-i 2 t[1+(4 m) \kappa]}, \\
\rho_{4 m+2}\left(\psi_{1}^{(\kappa)}, t\right) & =-\frac{(1-\kappa)[1+(4 m+1) \kappa]+2 t \kappa+i 2 t[1+(4 m+2) \kappa]}{(1-\kappa)[1+(4 m+1) \kappa]+2 t \kappa-i 2 t[1+(4 m+2) \kappa]}, \\
\rho_{4 m+3}\left(\psi_{1}^{(\kappa)}, t\right) & =-\frac{(1-\kappa)[1+(4 m+2-i) \kappa]+i 2 t[1+(4 m+3) \kappa]}{(1-\kappa)[1+(4 m+2+i) \kappa]-i 2 t[1+(4 m+3) \kappa]},
\end{aligned}
$$

for $m \geq 0, c_{1}\left(\psi_{1}^{(\kappa)}, t\right)=\kappa-2 t$,

$$
\begin{aligned}
& c_{4 m+2}\left(\psi_{1}^{(\kappa)}, t\right)=\frac{-\kappa}{1+4 m \kappa} \frac{(1-\kappa)^{2}[1+(4 m+1) \kappa]+4 t \kappa(1-\kappa)-4 t^{2}[1+(4 m-1) \kappa]}{(1-\kappa)^{2}[1+(4 m+1) \kappa]+4 t^{2}[1+(4 m-1) \kappa]}, \\
& c_{4 m+3}\left(\psi_{1}^{(\kappa)}, t\right)=\frac{-4 t \kappa}{1+(4 m+1) \kappa} \frac{(1-\kappa)[1+(4 m+1) \kappa]+2 t \kappa}{(1-\kappa)^{2}[1+(4 m+1) \kappa]+4 t \kappa(1-\kappa)+4 t^{2}[1+(4 m+1) \kappa]},
\end{aligned}
$$




$$
\begin{aligned}
& c_{4 m+4}\left(\psi_{1}^{(\kappa)}, t\right)=\frac{\kappa}{1+(4 m+2) \kappa} \frac{(1-\kappa)^{2}[1+(4 m+1) \kappa]+4 t \kappa(1-\kappa)-4 t^{2}[1+(4 m+3) \kappa]}{(1-\kappa)^{2}[1+(4 m+1) \kappa]+4 t^{2}[1+(4 m+3) \kappa]}, \\
& c_{4 m+5}\left(\psi_{1}^{(\kappa)}, t\right)=\frac{-2 \kappa(1-\kappa)}{1+(4 m+3) \kappa} \frac{(1-\kappa) \kappa-2 t[1+(4 m+3) \kappa]}{(1-\kappa)^{2}[1+(4 m+3) \kappa]-4 t \kappa(1-\kappa)+4 t^{2}[1+(4 m+3) \kappa]},
\end{aligned}
$$

for $m \geq 0$, and $\mathfrak{m}_{0}\left(\psi_{1}^{(\kappa)}, t\right)=0$,

$$
\begin{aligned}
\mathfrak{m}_{4 m+1}\left(\psi_{1}^{(\kappa)}, t\right)= & \frac{1+(4 m-1) \kappa}{2(1+(4 m) \kappa)^{2}} \\
& \times \frac{(1-\kappa)^{2}[1+(4 m+1) \kappa]^{2}+4 t \kappa(1-\kappa)[1+(4 m+1) \kappa]+4 t^{2}\left(\kappa^{2}+[1+(4 m) \kappa]^{2}\right)}{(1-\kappa)^{2}[1+(4 m+1) \kappa]+4 t^{2}[1+(4 m-1) \kappa]}, \\
\mathfrak{m}_{4 m+2}\left(\psi_{1}^{(\kappa)}, t\right)= & \frac{1+(4 m) \kappa}{2(1+(4 m+1) \kappa)^{2}} \\
& \times \frac{(1-\kappa)^{2}[1+(4 m+1) \kappa]^{2}+4 t \kappa(1-\kappa)[1+(4 m+1) \kappa]+4 t^{2}\left(\kappa^{2}+[1+(4 m+2) \kappa]^{2}\right)}{(1-\kappa)^{2}[1+(4 m+1) \kappa]+4 t \kappa(1-\kappa)+4 t^{2}[1+(4 m+1) \kappa]}, \\
\mathfrak{m}_{4 m+3}\left(\psi_{1}^{(\kappa)}, t\right)= & \frac{1+(4 m+1) \kappa}{2(1+(4 m+2) \kappa)^{2}} \\
& \times \frac{(1-\kappa)^{2}\left(\kappa^{2}+[1+(4 m+2) \kappa]^{2}\right)-4 t \kappa(1-\kappa)[1+(4 m+3) \kappa]+4 t^{2}[1+(4 m+3) \kappa]^{2}}{(1-\kappa)^{2}[1+(4 m+1) \kappa]+4 t^{2}[1+(4 m+3) \kappa]}, \\
\mathfrak{m}_{4 m+4}\left(\psi_{1}^{(\kappa)}, t\right)= & \frac{1+(4 m+2) \kappa}{2(1+(4 m+3) \kappa)^{2}} \\
& \times \frac{(1-\kappa)^{2}\left(\kappa^{2}+[1+(4 m+4) \kappa]^{2}\right)-4 t \kappa(1-\kappa)[1+(4 m+3) \kappa]+4 t^{2}[1+(4 m+3) \kappa]^{2}}{(1-\kappa)^{2}[1+(4 m+3) \kappa]-4 t \kappa(1-\kappa)+4 t^{2}[1+(4 m+3) \kappa]},
\end{aligned}
$$

for $m \geq 0$.

Theorem 6. Let the sequence $\left\{\rho_{n}\left(\psi_{1}^{(\kappa)}, t\right)\right\}_{n=0}^{\infty}$ be as in (16). Then for the polynomials $R_{n}\left(\psi_{1}^{(\kappa)}, t ; z\right), n \geq 0$, given by

$$
R_{n}\left(\psi_{1}^{(\kappa)}, t ; z\right)=\prod_{k=0}^{n-1} \frac{1+\overline{\rho_{k}\left(\psi_{1}^{(\kappa)}, t\right) \alpha_{k-1}\left(\psi_{1}^{(\kappa)}\right)}}{1+\mathcal{R e}\left[\rho_{k}\left(\psi_{1}^{(\kappa)}, t\right) \alpha_{k-1}\left(\psi_{1}^{(\kappa)}\right)\right]}\left[z S_{n-1}\left(\psi_{1}^{(\kappa)} ; z\right)-\rho_{n-1}\left(\psi_{1}^{(\kappa)}, t\right) S_{n-1}^{*}\left(\psi_{1}^{(\kappa)} ; z\right)\right],
$$

for $n \geq 1$, the following hold.

$$
R_{n+1}\left(\psi_{1}^{(\kappa)}, t ; z\right)=\left[\left(1+i c_{n+1}\left(\psi_{1}^{(\kappa)}, t\right)\right) z+\left(1-i c_{n+1}\left(\psi_{1}^{(\kappa)}, t\right)\right)\right] R_{n}\left(\psi_{1}^{(\kappa)}, t ; z\right)-4 d_{n+1}\left(\psi_{1}^{(\kappa)}, t\right) z R_{n-1}\left(\psi_{1}^{(\kappa)}, t ; z\right),
$$

for $n \geq 1$, with $R_{0}\left(\psi_{1}^{(\kappa)}, t ; z\right)=1$ and $R_{1}\left(\psi_{1}^{(\kappa)}, t ; z\right)=\left(1+i c_{1}\left(\psi_{1}^{(\kappa)}, t\right)\right) z+\left(1-i c_{1}\left(\psi_{1}^{(\kappa)}, t\right)\right)$, and

$$
\mathcal{N}^{(\kappa, t)}\left[z^{-n+j} R_{n}\left(\psi_{1}^{(\kappa)}, t ; z\right)\right]= \begin{cases}0, & 0 \leq j \leq n-1, \\ \gamma_{n}\left(\psi_{1}^{(\kappa)}, t\right), & j=n\end{cases}
$$

where $\gamma_{0}^{(\kappa)}(\lambda)=\frac{2 d_{1}}{1+i c_{1}\left(\psi_{1}^{(\kappa)}, t\right)}, \gamma_{n}\left(\psi_{1}^{(\kappa)}, t\right)=\frac{4 d_{n+1}\left(\psi_{1}^{(\kappa)}, t\right)}{1+i c_{n+1}\left(\psi_{1}^{(\kappa)}, t\right)} \gamma_{n-1}\left(\psi_{1}^{(\kappa)}, t\right), \quad n \geq 1$, and

$$
\mathcal{N}^{(\kappa, t)}[\ell]=\frac{4 d_{1}}{1+(\kappa-2 t)^{2}} f_{\mathcal{C}} \ell(\zeta) \frac{\zeta}{\zeta-1} d \psi_{1}^{(\kappa)}(\zeta)+i t \ell(1)
$$

Here, $d_{1} \neq 0,\left\{c_{n}\left(\psi_{1}^{(\kappa)}, t\right)\right\}_{n=1}^{\infty}$ and the minimal parameter sequence $\left\{\mathfrak{m}_{n}\left(\psi_{1}^{(\kappa)}, t\right)\right\}_{n=0}^{\infty}$ of the positive chain sequence $\left\{d_{n+1}\left(\psi_{1}^{(\kappa)}, t\right)\right\}_{n=1}^{\infty}$ are as in (17) and (18).

Since the integral $J=\int_{\mathcal{C}} \zeta(1-\zeta)^{-1}(\zeta-1)^{-1} d \psi_{1}^{(\kappa)}(\zeta)$ does not exist, the positive chain sequence $\left\{d_{n+1}\left(\psi_{1}^{(\kappa)}, t\right)\right\}_{n=1}^{\infty}$ is a SPPCS for all $t$. That is, $\left\{\mathfrak{m}_{n}\left(\psi_{1}^{(\kappa)}, t\right)\right\}_{n=0}^{\infty}$ is also the maximal parameter sequence of $\left\{d_{n+1}\left(\psi_{1}^{(\kappa)}, t\right)\right\}_{n=1}^{\infty}$.

\section{OPUC associated with the measures $\psi_{1}^{(\kappa)}\left(\lambda ;\right.$. ) and $\psi_{2}^{(\kappa)}$}

Results given in Theorem 1 also tell us that the Verblunsky coefficients $\alpha_{n}\left(\psi_{1}^{(\kappa)}(\lambda ;).\right)$ associated with the measure $\psi_{1}^{(\kappa)}(\lambda ;$. can be generated by

$$
\alpha_{n-1}\left(\psi_{1}^{(\kappa)}(\lambda ; .)\right)=\frac{1}{\rho_{n}\left(\psi_{1}^{(\kappa)}\right)} \frac{1-2 m_{n}^{(\kappa)}(\lambda)-i c_{n}^{(\kappa)}}{1+i c_{n}^{(\kappa)}}, \quad n \geq 1 .
$$


Here, $\left\{m_{n}^{(\kappa)}(\lambda)\right\}_{n=0}^{\infty}$ is the minimal parameter sequence of the positive chain sequence $\left\{d_{n}^{(\kappa)}(\lambda)\right\}_{n=1}^{\infty}$, where $d_{1}^{(\kappa)}(\lambda)=(1-\lambda) M_{1}^{(\kappa)}$ and $d_{n+1}^{(\kappa)}(\lambda)=d_{n+1}^{(\kappa)}, n \geq 1$. The values of $\left\{c_{n}^{(\kappa)}\right\}_{n=1}^{\infty}$ and the maximal parameter sequence $\left\{M_{n}^{(\kappa)}\right\}_{n=1}^{\infty}$ of the positive chain sequence $\left\{d_{n+1}^{(\kappa)}\right\}_{n=1}^{\infty}$ are explicitly provided in Theorem 5 .

Using the results given in Theorem 5, we can verify by induction that

$$
\begin{aligned}
& m_{4 m+1}^{(\kappa)}(\lambda)=\frac{\left[(4 m \kappa+1)^{2}+\kappa^{2}\right][(\kappa+4 m-1) \lambda+(1-\kappa)]}{2(4 m \kappa+1)\left[4 m\left(\kappa^{2}+4 m \kappa+1\right) \lambda+(4 m \kappa+1)(1-\kappa)\right]}, \\
& m_{4 m+2}^{(\kappa)}(\lambda)=\frac{\left[((4 m+2) \kappa+1)^{2}+\kappa^{2}\right]\left[4 m\left(\kappa^{2}+4 m \kappa+1\right) \lambda+(4 m \kappa+1)(1-\kappa)\right]}{2((4 m+1) \kappa+1)^{2}\left[\left((4 m+1) \kappa^{2}+4 m(4 m+2) \kappa+4 m+1\right) \lambda+((4 m+1) \kappa+1)(1-\kappa)\right]}, \\
& m_{4 m+3}^{(\kappa)}(\lambda)=\frac{[(4 m+3) \kappa+1]\left[\left((4 m+1) \kappa^{2}+4 m(4 m+2) \kappa+4 m+1\right) \lambda+((4 m+1) \kappa+1)(1-\kappa)\right]}{2((4 m+2) \kappa+1)\left[\left((4 m+2)\left(\kappa^{2}+(4 m+2) \kappa+1\right)\right) \lambda+((4 m+2) \kappa+1)(1-\kappa)\right]}, \\
& m_{4 m+4}^{(\kappa)}(\lambda)=\frac{\left((4 m+2)\left[\kappa^{2}+(4 m+2) \kappa+1\right]\right) \lambda+((4 m+2) \kappa+1)(1-\kappa)}{2((4 m+3) \kappa+1)[(\kappa+4 m+3) \lambda+(1-\kappa)]},
\end{aligned}
$$

for $m \geq 0$. Hence, by direct substitutions in (19) of the values of $m_{n}^{(\kappa)}(\lambda)$ presented above and $c_{n}^{(\kappa)}$ obtained in Theorem 5 , we can state the following.

Theorem 7. The Verblunsky coefficients associated with the measure $\psi_{1}^{(\kappa)}(\lambda ;$.) given in (13), for $0 \leq \kappa<1$ and for $0 \leq \lambda<1$, are

$$
\begin{aligned}
& \alpha_{4 m}\left(\psi_{1}^{(\kappa)}(\lambda ; .)\right)=\frac{-[(i(\kappa+4 m-1) \kappa-(4 m-1) \kappa-1) \lambda+i \kappa(1-\kappa)]}{\left(4 m\left[\kappa^{2}+4 m \kappa+1\right]\right) \lambda+(4 m \kappa+1)(1-\kappa)}, \\
& \alpha_{4 m+1}\left(\psi_{1}^{(\kappa)}(\lambda ; .)\right)=\frac{(1+i \kappa)^{2} \lambda-\kappa(1-\kappa)}{\left((4 m+1) \kappa^{2}+4 m(4 m+2) \kappa+4 m+1\right) \lambda+((4 m+1) \kappa+1)(1-\kappa)}, \\
& \alpha_{4 m+2}\left(\psi_{1}^{(\kappa)}(\lambda ; .)\right)=\frac{[i \kappa(\kappa+4 m+3)+(4 m+3) \kappa+1] \lambda+i \kappa(1-\kappa)}{\left[(4 m+2)\left(\kappa^{2}+(4 m+2) \kappa+1\right)\right] \lambda+[(4 m+2) \kappa+1](1-\kappa)}, \\
& \alpha_{4 m+3}\left(\psi_{1}^{(\kappa)}(\lambda ; .)\right)=\frac{\left[\kappa^{2}+2(4 m+3) \kappa+1\right] \lambda+\kappa(1-\kappa)}{[(4 m+3) \kappa+1][\kappa+4 m+3] \lambda+[(4 m+3) \kappa+1](1-\kappa)},
\end{aligned}
$$

for $m \geq 0$.

The choice $\lambda=1-\kappa$ establishes the following.

Corollary 7.1. The Verblunsky coefficients associated with the nontrivial probability measure $\tilde{\psi}_{1}^{(\kappa)}(z)=\psi_{1}^{(\kappa)}(1-\kappa ; z), 0<\kappa<1$, given by

$$
\int_{\mathcal{C}} f(\zeta) d \tilde{\psi}_{1}^{(\kappa)}(\zeta)=(1-\kappa) \kappa \int_{\mathcal{C}} f(\zeta) \frac{1}{2 \pi i \zeta} d \zeta+\kappa^{2} f(i)+(1-\kappa) f(1)
$$

are

$$
\begin{aligned}
& \alpha_{4 m}\left(\tilde{\psi}_{1}^{(\kappa)}\right)=\frac{(4 m-1) \kappa+1-i \kappa(\kappa+4 m)}{4 m \kappa^{2}+4 m(4 m \kappa+1)+4 m \kappa+1}, \\
& \alpha_{4 m+1}\left(\tilde{\psi}_{1}^{(\kappa)}\right)=\frac{(1+i \kappa)^{2}-\kappa}{(4 m+1) \kappa^{2}+[(4 m+1)(4 m+2)-1] \kappa+4 m+2}, \\
& \alpha_{4 m+2}\left(\tilde{\psi}_{1}^{(\kappa)}\right)=\frac{(4 m+3) \kappa+1+i \kappa(\kappa+4 m+4)}{(4 m+2) \kappa^{2}+(4 m+2)(4 m+3) \kappa+4 m+3}, \\
& \alpha_{4 m+3}\left(\tilde{\psi}_{1}^{(\kappa)}\right)=\frac{\kappa^{2}+[2(4 m+3)+1] \kappa+1}{(4 m+3) \kappa^{2}+[(4 m+3)(4 m+4)+1] \kappa+4 m+4},
\end{aligned}
$$

for $m \geq 0$.

The measure $\psi_{2}^{(\kappa)}$ given in (3) is such that the integral $J=\int_{\mathcal{C}} \zeta(1-\zeta)^{-1}(\zeta-1)^{-1} d \psi_{2}^{(\kappa)}(\zeta)$ exists, and hence, also the integral

$$
I=\int_{\mathcal{C}} \zeta(\zeta-1)^{-1} d \psi_{2}^{(\kappa)}(\zeta)=\int_{\mathcal{C}} \zeta(\zeta-1)^{-1} d \psi_{2}^{(\kappa)}(\zeta)
$$

exists. It is straightforward to verify that $I=(1-i \kappa) / 2$. Thus, we can state the following.

Theorem 8. The Verblunsky coefficients $\alpha_{n}\left(\psi_{2}^{(\kappa)}\right)$ associated with the measure $\psi_{2}^{(\kappa)}$, for $0 \leq \kappa<1$, are

$$
\alpha_{4 m}\left(\psi_{2}^{(\kappa)}\right)=-\frac{1-\kappa+i 2 \kappa}{4 m(4 m+3) \kappa+4 m+2},
$$




$$
\begin{aligned}
& \alpha_{4 m+1}\left(\psi_{2}^{(\kappa)}\right)=-\frac{(4 m+3) \kappa+1+i 4(m+1) \kappa}{\left[(4 m+2)^{2}+(4 m+1)\right] \kappa+4 m+3}, \\
& \alpha_{4 m+2}\left(\psi_{2}^{(\kappa)}\right)=-\frac{[2(4 m+3)+1] \kappa+1}{4(m+1)[(4 m+3) \kappa+1]}, \\
& \alpha_{4 m+3}\left(\psi_{2}^{(\kappa)}\right)=-\frac{(4 m+3) \kappa+1-i 4(m+1) \kappa}{\left[(4 m+5)^{2}-(4 m+6)\right] \kappa+4 m+5},
\end{aligned}
$$

for $m \geq 0$.

Proof. To obtain information about the Verblunsky coefficients associated with the measure $\psi_{2}^{(\kappa)}$, we start with the pair of real sequences $\left\{\left\{c_{n}^{(\kappa)}\right\}_{n=1}^{\infty},\left\{d_{n+1}^{(\kappa)}\right\}_{n=1}^{\infty}\right\}$ established in Theorem 5 .

The positive chain sequence $\left\{d_{n+1}^{(\kappa)}\right\}_{n=1}^{\infty}$ is expressed in terms of its maximal parameter sequence $\left\{M_{n+1}^{(\kappa)}\right\}_{n=0}^{\infty}$, which is explicitly provided in Theorem 5 . With the chain sequence $\left\{d_{n}^{(\kappa)}(0)\right\}_{n=1}^{\infty}$, where $d_{1}^{(\kappa)}(0)=(1-0) M_{1}^{(\kappa)}$ and $d_{n+1}^{(\kappa)}(0)=d_{n+1}^{(\kappa)}$, $n \geq 1$, we obtain from part b) of Theorem 1 the nontrivial probability measure $\psi_{1}^{(\kappa)}(z)=\psi_{1}^{(\kappa)}(0, z)$.

On the other hand, by part b) of Theorem 2 there exists a nontrivial probability measure $\phi$, where the respective Verblunsky coefficients satisfy

$$
\alpha_{n-1}(\phi)=\frac{1}{\rho_{n}^{(\kappa)}} \frac{1-2 \mathfrak{m}_{n}^{(\kappa)}-i c_{n+1}^{(\kappa)}}{1+i c_{n+1}^{(\kappa)}}, \quad n \geq 1
$$

Here, $\left\{\mathfrak{m}_{n}^{(\kappa)}\right\}_{n=0}^{\infty}$ is the minimal paramente sequence of $\left\{d_{n+1}^{(\kappa)}\right\}_{n=1}^{\infty}$ given by

$$
\mathfrak{m}_{0}^{(\kappa)}=0, \quad \mathfrak{m}_{n}^{(\kappa)}=\frac{d_{n+1}^{(\kappa)}}{1-\mathfrak{m}_{n-1}^{(\kappa)}}=\frac{\left(1-M_{n}^{(\kappa)}\right) M_{n+1}^{(\kappa)}}{1-\mathfrak{m}_{n-1}^{(\kappa)}}, \quad n \geq 1 .
$$

Thus, from the values of $M_{n}^{(\kappa)}$ given in Theorem 5 we obtain

$$
\begin{aligned}
\mathfrak{m}_{4 m+1}^{(\kappa)} & =\frac{1}{4} \frac{\left\{\left[(4 m+1)^{2}-(4 m+2)\right] \kappa+4 m+1\right\}\left[((4 m+2) \kappa+1)^{2}+\kappa^{2}\right]}{[2 m(4 m+3) \kappa+2 m+1][(4 m+1) \kappa+1]^{2}}, \\
\mathfrak{m}_{4 m+2}^{(\kappa)} & =\frac{[2 m(4 m+3) \kappa+2 m+1][(4 m+3) \kappa+1]}{\left\{\left[(4 m+2)^{2}+(4 m+1)\right] \kappa+4 m+3\right\}[(4 m+2) \kappa+1]}, \\
\mathfrak{m}_{4 m+3}^{(\kappa)} & =\frac{1}{8} \frac{\left[(4 m+2)^{2}+(4 m+1)\right] \kappa+4 m+3}{(m+1)[(4 m+3) \kappa+1]}, \\
\mathfrak{m}_{4 m+4}^{(\kappa)} & =\frac{2(m+1)\left[((4 m+4) \kappa+1)^{2}+\kappa^{2}\right]}{\left\{\left[(4 m+5)^{2}-(4 m+6)\right] \kappa+4 m+5\right\}[(4 m+4) \kappa+1]},
\end{aligned}
$$

for $m \geq 0$. The above explicit expressions for $\mathfrak{m}_{n}^{(\kappa)}$ are easily verified by induction.

The sequence $\left\{\rho_{n}^{(\kappa)}\right\}_{n=0}^{\infty}$ in $(21)$ is such that

$$
\rho_{0}^{(\kappa)}=-\frac{\left(1-i c_{1}^{(\kappa)}\right)}{\left(1+i c_{1}^{(\kappa)}\right)} \quad \text { and } \quad \rho_{n}^{(\kappa)}=\frac{\left(1-i c_{n+1}^{(\kappa)}\right)}{\left(1+i c_{n+1}^{(\kappa)}\right)} \rho_{n-1}^{(\kappa)}, \quad n \geq 1
$$

Hence, from (15) $\rho_{n}^{(\kappa)}=-\rho_{n+1}\left(\psi_{1}^{(\kappa)}\right), n \geq 0$, are also explicitly known. Thus, by direct substitution of the values of $\left\{\mathfrak{m}_{n}^{(\kappa)}\right\}_{n=0}^{\infty}$ and $\left\{\rho_{n}^{(\kappa)}\right\}_{n=0}^{\infty}$ in (21) we find the values of $\alpha_{n}(\phi), n \geq 0$, which are exactly the values found in the present theorem. Finally to verify that $\phi(z)=\psi_{2}^{(\kappa)}(z)$, we use Theorem 3 .

From results presented immediately after Theorem 2 , it is easily seen that $\rho_{0}^{(\kappa)}=\tau=-I / \bar{I}$, where the value of the integral $I$ for the measure $\psi_{2}^{(\kappa)}$ is established in (20). Therefore, in terms of the notation adopted in Theorem 2, we can write $\rho_{n}^{(\kappa)}=$ $\rho_{n}\left(\psi_{2}^{(\kappa)}, \tau(0)\right), n \geq 0$.

However, we can generate other sequences of para-orthogonal polynomials

$$
R_{n}\left(\psi_{2}^{(\kappa)}, \tau(t) ; z\right)=\prod_{k=0}^{n-1} \frac{1+\overline{\rho_{k}\left(\psi_{2}^{(\kappa)}, \tau(t)\right) \alpha_{k-1}\left(\psi_{2}^{(\kappa)}\right)}}{1+\mathcal{R e}\left[\rho_{k}\left(\psi_{2}^{(\kappa)}, \tau(t)\right) \alpha_{k-1}\left(\psi_{2}^{(\kappa)}\right)\right]}\left[z S_{n-1}\left(\psi_{2}^{(\kappa)} ; z\right)-\rho_{n-1}\left(\psi_{2}^{(\kappa)}, \tau(t)\right) S_{n-1}^{*}\left(\psi_{2}^{(\kappa)} ; z\right)\right],
$$

from Theorem 2 using the sequence $\left\{\rho_{n}\left(\psi_{2}^{(\kappa)}, \tau(t)\right)\right\}_{n=0}^{\infty}$ given by

$$
\rho_{0}\left(\psi_{2}^{(\kappa)}, \tau(t)\right)=-\frac{I+i t}{\bar{I}-i t} \quad \text { and } \quad \rho_{n}\left(\psi_{2}^{(\kappa)}, \tau(t)\right)=\frac{\rho_{n-1}\left(\psi_{2}^{(\kappa)}, \tau(t)\right)-\overline{\alpha_{n-1}\left(\psi_{2}^{(\kappa)}\right)}}{1-\rho_{n-1}\left(\psi_{2}^{(\kappa)}, \tau(t)\right) \alpha_{n-1}\left(\psi_{2}^{(\kappa)}\right)}, \quad n \geq 1
$$




\section{Acknowledgment}

We would like to thank the referee for providing us with many constructive comments and suggestions.

The first and third authors are supported by funds from CNPq (\#401891/2013-5, \#475502/2013-2) and FAPESP (\#09/13832-9, \#14/22571-2) of Brazil. The second author is supported by grant from CAPES of Brazil.

\section{References}

[1] C. F. Bracciali, A. Sri Ranga, A. Swaminathan, Para-orthogonal polynomials on the unit circle satisfying three term recurrence formulas, submitted (see arXiv:1406.0719v1 [math.CA]).

[2] M.J. Cantero, L. Moral, L. Velázquez, Measures and para-orthogonal polynomials on the unit circle, East J. Approx. 8 (2002) $447-464$

[3] K. Castillo, M.S. Costa, A. Sri Ranga, D.O. Veronese, A Favard type theorem for orthogonal polynomials on the unit circle from a three term recurrence formula, J. Approx. Theory 184 (2014) 146-162.

[4] T.S. Chihara, An introduction to orthogonal polynomials, in: Mathematics and its Applications Series, Gordon and Breach, New York, 1978.

[5] M.S. Costa, H.M. Felix, A. Sri Ranga, Orthogonal polynomials on the unit circle and chain sequences, J. Approx. Theory 173 (2013) 14-32.

[6] L. Golinskii, Quadrature formula and zeros of para-orthogonal polynomials on the unit circle, Acta Math. Hung. 96 (2002) 169-186.

[7] M.E.H. Ismail, Classical and quantum orthogonal polynomials in one variable, in: Encyclopedia of Mathematics and its Applications, 98, Cambridge University Press, Cambridge, 2005.

[8] B. Simon, Orthogonal polynomials on the unit circle. Part 1. Classical theory, in: Part 1, American Mathematical Society, 54, American Mathematical Society Colloquium Publications, Providence, RI, 2005a.

[9] B. Simon, Orthogonal polynomials on the unit circle. Part 2. Spectral theory, in: Part 2, American Mathematical Society, 54, American Mathematical Society Colloquium Publications, Providence, RI, 2005b.

[10] B. Simon, Szegö's Theorem and its Descendants: Spectral Theory for $l^{2}$ Perturbations of Orthogonal Polynomials, Princeton University Press, Princeton, 2011.

[11] G. Szegő, Orthogonal polynomials, in: Amer. Math. Soc., Fourth Ed., 23, American Mathematical Society Colloquium Publications, Providence, RI, 1975.

[12] D.S. Watkins, Fundamentals of Matrix Computations, John Wiley \& Sons, 1991.

[13] M.L. Wong, First and second kind paraorthogonal polynomials and their zeros, J. Approx. Theory 146 (2007) 282-293. 